\title{
High-latitude magnetic fields and their time derivatives: interhemispheric similarities
}

\author{
Ari Viljanen ${ }^{1}$ and Eija Tanskanen ${ }^{1,2}$ \\ ${ }^{1}$ Finnish Meteorological Institute, P.O.B. 503, FI-00101 Helsinki, Finland \\ ${ }^{2}$ University of Bergen, Dept. of Physics and Technology, Allégatan 55, Bergen N-5007, Norway
}

(Received September 15, 2011; Revised May 8, 2012; Accepted May 18, 2012; Online published February 19, 2013)

\begin{abstract}
There are two simple and objective measures of geomagnetic activity: the daily range of field components, and the daily maximum of the time derivative of the field. We study these using data from the geomagnetic latitude range of 50-85 deg, covering both the northern and southern hemispheres in 2003-2005. These activity indicators reach their maximum around the magnetic latitude of $70 \mathrm{deg}$. When comparing northern and southern sites of approximately equal geomagnetic latitudes, there are, in general, no striking interhemispheric differences.
\end{abstract}

Key words: Geomagnetic activity, interhemispheric comparisons.

\section{Introduction}

The dipole axis of the geomagnetic field is tilted from the Sun-Earth line which causes seasonal variations and hemispherical asymmetries attributed to the solar radiation, and the solar wind, input into the magnetosphere. Studies of the high-latitude ionosphere have previously been concentrated on the northern hemisphere, due to a better coverage of ground-based observations. Ground-based magnetometers play a central role, since they provide long-term continuous and homogeneous data sets. An increasing number of facilities have been installed in the Antarctic, which makes it now possible to perform extensive interhemispheric comparisons.

Previous statistical investigations based on high-latitude ground magnetometer recordings simultaneously in both hemispheres have been quite rare and limited by spatial and temporal coverage. For example, Hajkowicz (2006) considered the largest negative value of the daily horizontal field at Macquarie Island in the southern hemisphere and at six auroral and subauroral sites in the northern hemisphere in 2003 for 193 disturbed days.

Lepidi et al. (2011) investigated the diurnal variation during the quiet year 2006 as observed at six polar observatories, three in the northern, and three in the southern, hemisphere in the geomagnetic latitude range of 77-89 deg. The average daily curves of the geographic north and east field components are very similar at corresponding sites in both hemispheres, except for a sign reversal in the east component between north and south.

Posch et al. (1999) studied the characteristics of broadband ULF magnetic pulsations at two Arctic stations and their conjugate stations at the South Pole. They found that there was a substantial fraction of days during which there

Copyright (C) The Society of Geomagnetism and Earth, Planetary and Space Sciences (SGEPSS); The Seismological Society of Japan; The Volcanological Society of Japan; The Geodetic Society of Japan; The Japanese Society for Planetary Sciences; TERRAPUB.

doi:10.5047/eps.2012.05.014 was a significant temporal disagreement between the hemispheres, but the solar wind velocity still appears to control the overall daily intensity.

Lyatskaya et al. (2008) considered the correlation between the AL index (showing substorm activity in the northern hemisphere), and the Polar Cap (PC) and Polar Magnetic (PM) indices measuring magnetic activity in the polar caps. Using the data of four years (1990-91, 1997-98) they found that while, in the northern winter, the correlation between AL and the northern PC/PM indices is very good, in the northern summer, AL correlates much better with the southern PC/PM indices. In other words, substorm activity in the summer correlates much better with the geomagnetic activity in the opposite polar cap. They suggested that this effect may be caused by interhemispheric field-aligned currents flowing from the summer high-latitude ionosphere and closing through the ionosphere in the opposite auroral zone.

Weygand and Zesta (2008) compared the standard auroral electrojet (AE) index to the southern AE (SAE) index derived from the data of seven stations located in the southern auroral region during 3-10 December, 2005. They also constructed a northern AE index (NAE) of the stations conjugate to the southern sites. They found that many of the differences between the SAE and the standard AE can be explained by the gap in the southern magnetometer array. However, they also found significant north-south asymmetries that were not related to this gap. They suggested that the asymmetries likely result from interhemispheric asymmetries a rising under a strongly southward interplanetary magnetic field (IMF), or when the IMF $B_{y}$ is large. Interhemispheric asymmetries in ionospheric conductivity and local ground-conductivity anomalies may influence the signals in the magnetometers and may be a source of the SAE vs. NAE differences.

Polar-orbit satellites at low altitudes provide a uniform spatial coverage of observations from both hemispheres, although they cannot yield a uniform time series at a fixed lo- 
Table 1. Stations used in this study. Corrected geomagnetic coordinates (CGM) are determined for the year 2005 with the online service http://omniweb.gsfc.nasa.gov/vitmo/cgm.html. The last column shows the coverage of data in 2003-2005.

\begin{tabular}{|c|c|c|c|c|}
\hline Code & CGM lat & CGM long & Oval angle & $\operatorname{Cov}(\%)$ \\
\hline THL & 84.97 & 29.68 & -32.76 & 100 \\
\hline RES & 83.06 & 321.63 & 19.96 & 100 \\
\hline NAL & 76.30 & 110.54 & -22.67 & 94 \\
\hline GDH & 75.40 & 38.93 & -25.69 & 100 \\
\hline LYR & 75.35 & 111.45 & -19.82 & 95 \\
\hline HOR & 74.23 & 108.99 & -17.85 & 99 \\
\hline HOP & 73.18 & 114.64 & -14.04 & 55 \\
\hline BJN & 71.55 & 107.58 & -12.95 & 79 \\
\hline YKC & 69.36 & 301.48 & 22.82 & 95 \\
\hline SOR & 67.44 & 105.80 & -8.63 & 96 \\
\hline TRO & 66.72 & 102.52 & -8.82 & 99 \\
\hline AND & 66.52 & 99.97 & -9.32 & 99 \\
\hline KEV & 66.43 & 108.93 & -6.94 & 97 \\
\hline MAS & 66.28 & 106.08 & -7.46 & 91 \\
\hline KIL & 65.97 & 103.45 & -7.88 & 94 \\
\hline LEK & 65.46 & 97.10 & -9.14 & 95 \\
\hline $\mathrm{ABK}$ & 65.38 & 101.40 & -7.92 & 97 \\
\hline IVA & 65.21 & 108.28 & -6.11 & 93 \\
\hline MUO & 64.82 & 104.90 & -6.61 & 84 \\
\hline KIR & 64.78 & 102.30 & -7.22 & 98 \\
\hline SOD & 64.03 & 106.99 & -5.59 & 100 \\
\hline PEL & 63.65 & 104.63 & -5.86 & 85 \\
\hline RVK & 62.28 & 92.95 & -7.90 & 95 \\
\hline OUJ & 61.09 & 105.91 & -4.10 & 93 \\
\hline DOB & 59.32 & 89.87 & -6.87 & 99 \\
\hline HAN & 58.82 & 104.41 & -3.28 & 90 \\
\hline NUR & 56.99 & 101.98 & -2.91 & 100 \\
\hline UPS & 56.58 & 95.60 & -3.95 & 94 \\
\hline KAR & 56.42 & 85.39 & -6.47 & 66 \\
\hline TAR & 54.58 & 102.72 & -1.80 & 96 \\
\hline EYR & -50.13 & 256.57 & 14.84 & 100 \\
\hline AIA & -50.34 & 9.14 & -3.57 & 100 \\
\hline CZT & -53.28 & 106.21 & -14.66 & 99 \\
\hline PAF & -58.61 & 122.38 & -18.08 & 100 \\
\hline MCQ & -64.35 & 248.32 & 18.68 & 98 \\
\hline MAW & -70.32 & 90.31 & -46.08 & 98 \\
\hline SBA & -79.94 & 327.26 & 89.06 & 98 \\
\hline DRV & -80.45 & 236.00 & 25.26 & 94 \\
\hline CSY & -80.78 & 157.02 & -22.77 & 99 \\
\hline
\end{tabular}

cation. However, spacecraft recordings of sufficient length give a reliable average description, as shown by Juusola et al. (2009), who studied five years of CHAMP data to determine auroral ionospheric currents (altitude adjusted corrected geomagnetic latitudes 55-76.5 deg). They included about 20000 overflights across the northern auroral region and about 24000 in the south.

We will consider geomagnetic variations using data from a large number of Arctic and Antarctic magnetometer stations. We focus on investigating the daily level of activity of field variations and of the time derivative of the field. The main objective is to find out whether there are differences in the average activity levels between the hemispheres. Compared with previous studies, we will use a much more extensive set of ground magnetometer stations over a wide latitude range.

\section{Data and Methods}

In interhemispheric comparisons, it is crucial to use geomagnetically conjugate sites connected by the same field line. Conjugacy is typically determined with statistical models describing the average field topology in the nearspace. As noted by Kozyreva et al. (2006), the concept of conjugacy makes sense only in a closed magnetosphere. In the polar caps that are projected on the geomagnetic field open lines, it is more logical to use the term symmetry, referring to the corresponding geomagnetic latitudes and longitudes calculated according to a selected field model.

We use data from 30 northern hemisphere magnetometer stations and from 9 southern hemisphere locations (Table 1). The active years of 2003-2005, during the declining solar cycle phase, are considered. We have used 1minute data, and assumed that the daily average field yields 

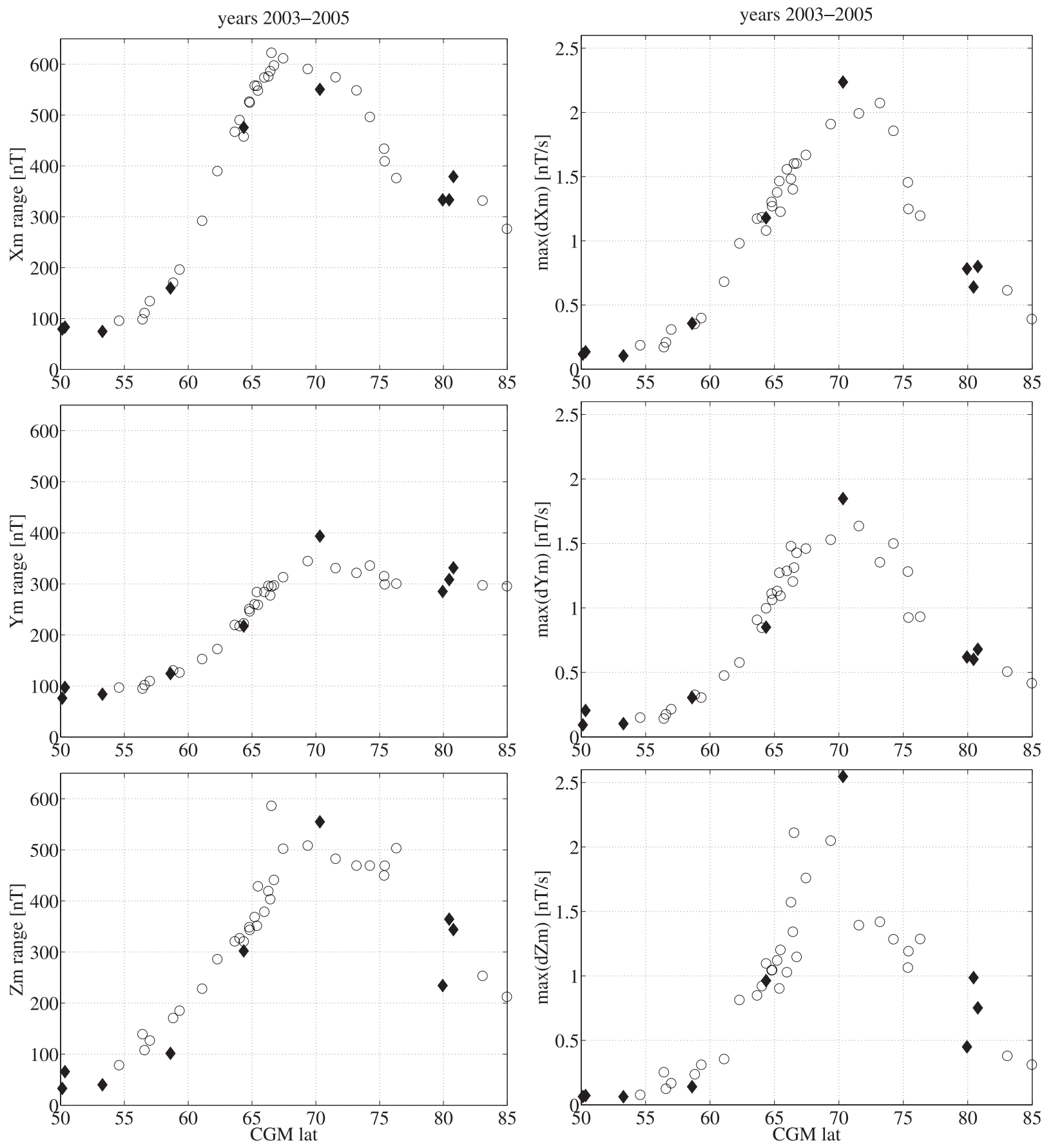

Fig. 1. Left: average daily range of the magnetic field at high-latitude stations. Right: average daily maximum of the time derivative of the magnetic field. From top to bottom: magnetic north, east and downward components. Open circles: northern hemisphere, filled diamonds: southern hemisphere.

a reasonable quiet time baseline. The north and east components $\left(X_{m}, Y_{m}\right)$ of the magnetic field in the corrected geomagnetic (CGM) coordinate frame are converted from the baseline-subtracted geographic components $(X, Y)$ according to

$$
X_{m}=X \cos \alpha+Y \sin \alpha, \quad Y_{m}=-X \sin \alpha+Y \cos \alpha .
$$

The downward field component is the same in both geographic and CGM coordinates $\left(Z_{m}=Z\right)$. We will also consider the magnitude of the horizontal magnetic field,

$$
|\mathbf{H}|=\sqrt{X^{2}+Y^{2}}=\sqrt{X_{m}^{2}+Y_{m}^{2}},
$$

which is independent of the coordinate system. The oval angle $\alpha$ in Eq. (1) is the angle between the local tangents to the CGM and geographic latitudes. In the northern hemisphere, this angle is presented as the azimuth to the local magnetic north if the eastward tangent to the CGM latitude points southward from local east, measured positive to east. Correspondingly, in the southern hemisphere, it is presented as the azimuth to the local magnetic south if the westward tangent to the CGM latitude points northward from local west, measured positive to west.

In addition, to the variation field, we will study its time derivative, which is calculated as the difference in the field 
between successive 1-minute time steps. The time derivative provides a different viewpoint from the variation field, especially when geomagnetically-induced currents are of interest (Viljanen et al., 2001, 2006; Trichtchenko and Boteler, 2004).

We characterise magnetic activity by the daily range of the field components and by the daily maximum of $|\mathbf{H}|$ and of the time derivatives. Except for $|\mathbf{H}|$, these measurements do not depend on the selection of the quiet time baseline. When needed, the baseline was determined by subtracting the daily average.

\section{Results}

The magnetic variation field $\left(X_{m}, Y_{m}, Z_{m}\right)$ (Fig. 1, lefthand-side plots) shows a clear increase from subauroral latitudes $(50 \mathrm{deg}$ ) to the auroral region $(70 \mathrm{deg})$, most prominently in $X_{m}$ and $Z_{m}$. The horizontal components decrease to nearly a constant level of $300 \mathrm{nT}$ at high latitudes. $X_{m}$ and $Y_{m}$ are equally large at subauroral and high latitudes, and $X_{m}$ dominates in the auroral region.

All components of the time derivative of the magnetic field (Fig. 1, right-hand-side plots) increase from subauroral latitudes to the auroral region. Contrary to the variation field, there is a rapid decrease of all components at high latitudes. Also, as opposed to the variation field, all components have quite comparable magnitudes at all latitudes. We note that especially the vertical component $\left(Z_{m}\right)$ is evidently affected by local conductivity anomalies in the ground. If a station is located above a highly-conducting structure, then the vertical field variations tend to be smaller than in the surroundings.

When the field variation and its time derivative are compared (Fig. 2), the maximum of the horizontal variation $|\mathbf{H}|$ occurs at about $68 \mathrm{deg}$, whereas $|d \mathbf{H} / d t|$ reaches its maximum at $72 \mathrm{deg}$ (excluding station MAW at $70 \mathrm{deg}$ ). We also note a large variability of the variations as indicated by the standard deviations.

Hajkowicz (2006) found that the latitudinal profile of the daily horizontal field $(\Delta H)$ for each season reaches a maximum at about $65 \mathrm{deg}$ (CGM). This is different from our result indicating that the maximum takes place around $68 \mathrm{deg}$ (Fig. 2). Although we have considered the daily maximum of $(|\mathbf{H}|)$, in practice it is close to $\Delta H$ in the auroral region, since $\left|X_{m}\right|$ is clearly larger than $\left|Y_{m}\right|$ there due to the dominance of the westward electrojet during the most disturbed times.

Ahn et al. (2005) investigated the statistics of westward electrojets (WEJ), related to negative $X$ variations, in 1998 in the northern hemisphere. For variations of a few hundred $\mathrm{nT}$, they found that WEJ was centered at about latitudes of 66-68 deg. This is consistent with our findings in Fig. 2. Although Ahn et al. (2005) restricted their study to the WEJ, it is likely that the daily maximum of $|\mathbf{H}|$ used by us is also related to the WEJ in most cases, since the highest magnetic activity is typically associated with them.

The location of the maximum $|d \mathbf{H} / d t|$ obviously depends on two major factors: substorm activations before midnight and morning-time pulsations, as discussed by Viljanen et al. (2001). Concerning especially Pc5 pulsations (periods 150-600 s), their latitudinal occurrence peaks
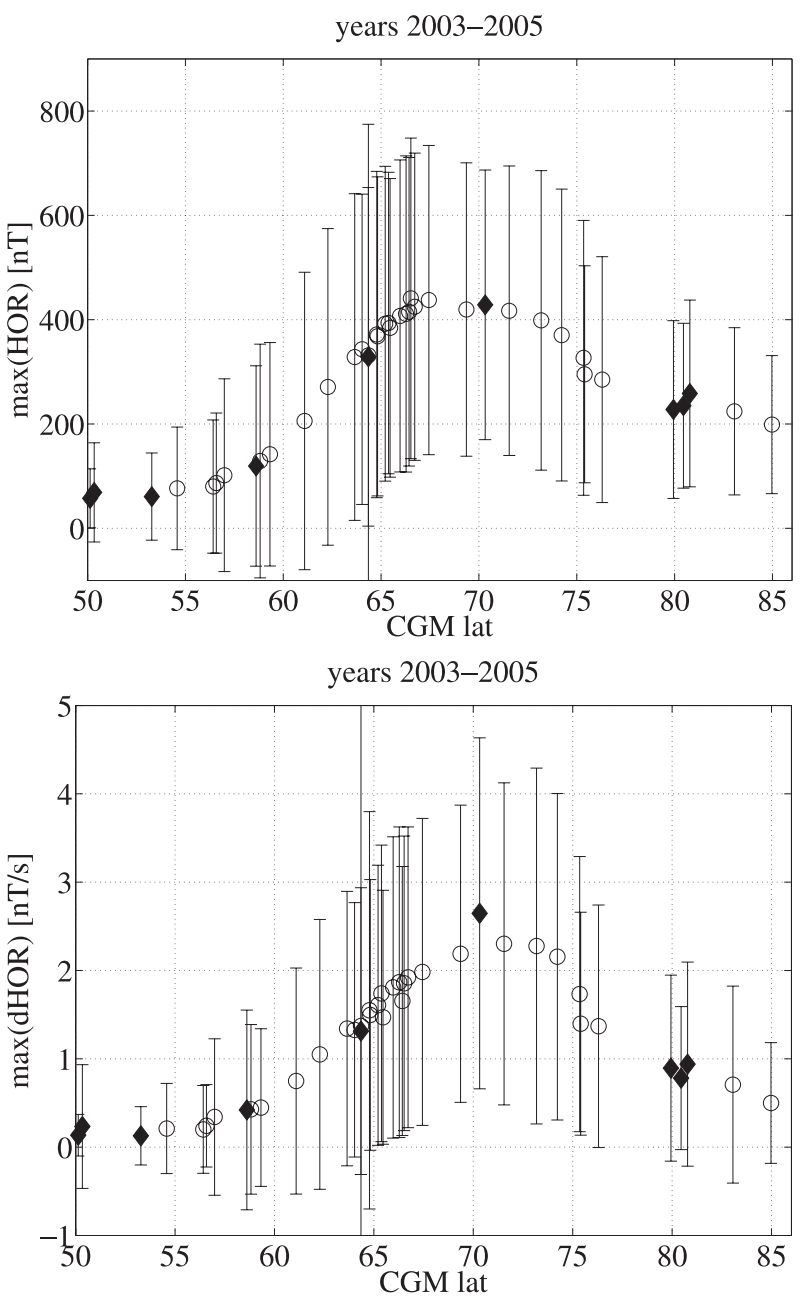

Fig. 2. Upper panel: Average of the daily maximum of the horizontal magnetic field vector $(\mathbf{H})$ at high-latitude stations. Lower panel: Average of the daily maximum of the time derivative of the horizontal magnetic field vector $(d \mathbf{H} / d t)$. Open circles: northern hemisphere, filled diamonds: southern hemisphere. Error bars correspond to the standard deviations.

around 70 degrees (Baker et al., 2003). To distinguish, more quantitatively, different drivers of rapid variations, one possibility could be to study different MLT periods separately, for example 16-02 (dominated by electrojets), 02-12 (Pc5) and 12-16 (peak time of Pc1-2; see Plyasova-Bakounina et al., 1996). Using simultaneous solar wind data would also be necessary to investigate the role of magnetosphericdriven field-line resonances and of driving caused directly by solar wind forcing on open field lines.

Viljanen et al. (2006) considered the behaviour of $|d \mathbf{H} / d t|$ during substorms in northern Europe. Their figure 2 shows that the location of the average maximum of $|d \mathbf{H} / d t|$ depends on whether the storm-time $\left(D_{\text {st }}<-40\right.$ $\mathrm{nT})$, or non-storm-time $\left(D_{\text {st }}>-40 \mathrm{nT}\right)$, substorms are studied. For the former, the maximum occurs at about 67 deg magnetic north, whereas for the latter, it is located at about $72 \mathrm{deg}$. These values are not directly comparable to the present study, since we have included, here, all the data, and we did not try to classify the type of event associated with each maximum of $|d \mathbf{H} / d t|$. However, Fig. 2 is gen- 
erally consistent with the previous results showing maximum variations around the $70 \mathrm{deg}$ latitude. Boteler (2001) presented a probability map of the occurrence of an hour with $d B / d t \geq 300 \mathrm{nT} / \mathrm{min}(5 \mathrm{nT} / \mathrm{s})$ in Canada. He found the maximum probability in the magnetic latitude range of about 66-69 deg. This is a few degrees south of the maximum found in our study. However, the measure of activity is different from ours, and Boteler (2001) considered rare events with a high threshold of $d B / d t$.

Station MAW (CGM lat 70.32 deg south) shows some peculiar features, especially in the time derivative. It has the largest average of $|d \mathbf{H} / d t|$, although $|\mathbf{H}|$ has an equal magnitude to other sites of nearly the same geomagnetic latitude (Fig. 2). When considered separately, $X$ and $Y$ components at MAW differ from the general behaviour: $X$ is smaller and $Y$ larger than could be expected. This may be due to a nearby highly-conducting anomaly in the ground, but without further knowledge of the local geology a definite explanation is not possible.

Finally, we note that extremely large variations show a different latitudinal behaviour than the averaged one. Thomson et al. (2011) studied the largest time derivatives and deviations from the quiet-time level of the horizontal field in Europe. They found the maximum values between 55-60 deg in magnetic latitudes. This is about 10 degrees south of the maximum shown in Fig. 2. As Thomson et al. (2011) note, this can be attributed to an enhanced auroral electrojet moving equatorward under strong solar wind forcing, consistent with the results of Rostoker and Phan (1986) and Ahn et al. (2005).

\section{Discussion}

The overall behaviour of the magnetic field and its time derivative is as expected: the daily range of all components and the time derivatives reach their maximum in the auroral region around the magnetic latitude of $70 \mathrm{deg}$. In general, when comparing sites of approximately equal geomagnetic latitudes, there are no striking differences between the northern and southern hemispheres, although a few stations show some anomalous behaviour. Such deviations are most probably due to the secondary effect of induced currents in the ground, and not due to ionospheric conditions. The similarity of the hemispheres in a statistical sense also agrees very well with the results by Juusola et al. (2009) concerning ionospheric currents determined by magnetometer recordings onboard a low-orbit satellite. Finally, we emphasise that, although the long-term average features of field variations and their time derivatives look quite similar in both hemispheres, there are significant differences when single events are considered (Posch et al., 1999; Papitashvili et al., 2000; Hajkowicz, 2006; Kozyreva et al., 2006).
Acknowledgments. Geomagnetic data used in this study were obtained from the World Data Centre for Geomagnetism (Edinburgh) and from the IMAGE magnetometer network. The work of E.T. was supported by the Academy of Finland (project no. 121289).

\section{References}

Ahn, B.-H., G. X. Chen, W. Sun, J. W. Gjerloev, Y. Kamide, J. B. Sigwarth, and L. A. Frank, Equatorward expansion of the westward electrojet during magnetically disturbed periods, J. Geophys. Res., 110, doi:10.1029/2004JA010553, 2005.

Baker, G., E. F. Donovan, and B. J. Jackel, A comprehensive survey of auroral latitude Pc5 pulsation characteristics, J. Geophys. Res., 108(A10), 1384, doi:10.1029/2002JA009801, 2003.

Boteler, D. H., Assessment of geomagnetic hazard to power systems in Canada, Nat. Haz., 23, 101-120, 2001.

Hajkowicz, L. A., Magnetoconjugate phenomena in Alaska and Macquarie Is., Australia in 2003: Position of the global maximum iso-aurorae, Ann. Geophys., 24, 2611-2617, 2006.

Juusola, L., K. Kauristie, O. Amm, and P. Ritter, Statistical dependence of auroral ionospheric currents on solar wind and geomagnetic parameters from 5 years of CHAMP satellite data, Ann. Geophys., 27, 1005-1017, www.ann-geophys.net/27/1005/2009/, 2009.

Kozyreva, O. V., N. G. Kleimenova, A. E. Levitin, and J. Watermann, Long-period geomagnetic pulsations in the quasi-conjugate arctic and antarctic regions during the magnetic storm of April 16-17, 1999, Geomagn. Aeron., 46, 622-634, 2006.

Lepidi, S., L. Cafarella, M. Pietrolungo, and D. Di Mauro, Daily variation characteristics at polar geomagnetic observatories, Adv. Space Res., 48, 521-528, 2011.

Lyatskaya, S., W. Lyatsky, and G. V. Khazanov, Relationship between substorm activity and magnetic disturbances in two polar caps, Geophys. Res. Lett., 35, L20104, doi:10.1029/2008GL035187, 2008.

Papitashvili, V. O., C. R. Clauer, F. Christiansen, V. A. Pilipenko, V. A. Popov, O. Rasmussen, V. P. Suchdeo, and J. F. Watermann, Geomagnetic disturbances at high latitudes during very low solar wind density event, Geophys. Res. Lett., 27(23), 3785-3788, doi:10.1029/2000GL000089, 2000.

Plyasova-Bakounina, T., J. Kangas, K. Mursula, O. Molchanov, and A. Green, Pc 1-2 and Pc 4-5 pulsations observed at a network of highlatitude stations, J. Geophys. Res., 101(A5), doi:10.1029/95JA03770, 1996.

Posch, J. L., M. J. Engebretson, A. T. Weatherwax, D. L. Detrick, W. J. Hughes, and C. G. Maclennan, Characteristics of broadband ULF magnetic pulsations at conjugate cusp latitude stations, J. Geophys. Res., 104(A1), 311-331, doi:10.1029/98JA02722, 1999.

Rostoker, G. and T. D. Phan, Variation of auroral electrojet spatial location as a function of the level of magnetospheric activity, J. Geophys. Res., 91, 1716-1722, 1986.

Thomson, A., E. Dawson, and S. Reay, Quantifying extreme behaviour in geomagnetic activity, Space Weather, 9, S10001, doi:10.1029/2011SW000696, 2011.

Trichtchenko, L. and D. H. Boteler, Modeling geomagnetically induced currents using geomagnetic indices and data, IEEE T. Plasma Sci., 32, 1459-1467, 2004.

Viljanen, A., H. Nevanlinna, K. Pajunpää, and A. Pulkkinen, Time derivative of the horizontal geomagnetic field as an activity indicator, Ann. Geophys., 19, 1107-1118, 2001.

Viljanen, A., E. I. Tanskanen, and A. Pulkkinen, Relation between substorm characteristics and rapid temporal variations of the ground magnetic field, Ann. Geophys., 24, 725-733, 2006.

Weygand, J. M. and E. Zesta, Comparison of auroral electrojet indices in the Northern and Southern Hemispheres, J. Geophys. Res., 113, A08202, doi:10.1029/2008JA013055, 2008.

A. Viljanen (e-mail: ari.viljanen@fmi.fi) and E. Tanskanen 